\title{
A Phase II Study of Sagopilone (ZK 219477; ZK-EPO) in Patients With Breast Cancer and Brain Metastases
}

\author{
Rachel A. Freedman ${ }^{1}$, Elizabeth Bullitt ${ }^{2}$, Lixian Sun ${ }^{3}$, Rebecca Gelman ${ }^{3}$, Gordon Harris ${ }^{4}$, \\ Jennifer A. Ligibel ${ }^{1}$, lan E. Krop ${ }^{1}$, Ann H. Partridge ${ }^{1}$, Emily Eisenberg ${ }^{1}$, Eric P. Winer ${ }^{1}$, and \\ Nancy U. Lin ${ }^{1}$ \\ ${ }^{1}$ Department of Medical Oncology, Dana-Farber Cancer Institute, Harvard Medical School, \\ Boston, MA \\ ${ }^{2}$ Department of Neurosurgery, University of North Carolina, Chapel Hill, NC \\ ${ }^{3}$ Department of Biostatistics \& Computational Biology, Dana-Farber Cancer Institute, Boston, MA \\ ${ }^{4}$ Department of Radiology, Massachusetts General Hospital, Harvard Medical School, Boston, \\ MA
}

\begin{abstract}
Treatments for women with recurrent brain metastases from breast cancer are limited. In this phase II study, we administered sagopilone to patients with breast cancer and brain metastases. We observed modest activity with a central nervous system objective response rate of $13.3 \%$; however, median PFS was disappointing. Further studies should focus on other agents to treat this challenging clinical problem.
\end{abstract}

Background-Patients with progressive metastatic breast cancer to the central nervous system (CNS) have limited treatment options.

Patients and Methods-We conducted a phase II study of sagopilone, an epothilone B analogue that crosses the blood-brain barrier, in patients with breast cancer brain metastases. Women were treated with $16 \mathrm{mg} / \mathrm{m}^{2}$ or $22 \mathrm{mg} / \mathrm{m}^{2}$ intravenously every 21 days. The primary endpoint was CNS objective response rate (ORR). Secondary endpoints included toxicity, progression-free survival (PFS), and overall survival (OS). Using modified, high-resolution magnetic resonance angiography (MRA), we also evaluated changes in vessel tortuosity with treatment.

Results-Fifteen women were enrolled; all had progressive CNS disease despite whole-brain radiotherapy. Two patients achieved a partial response (ORR, 13.3\%) and remained in the study for 6 cycles. Responses were not associated with normalization of tumor-associated vessels on correlative imaging studies. Median PFS and OS were 1.4 months and 5.3 months, respectively. The most common grade 3 toxicities were lymphopenia and fatigue. Enrollment was stopped prematurely because of limited observed activity and slow accrual.

Conclusions-Sagopilone was associated with modest CNS activity in patients with breast cancer; however median PFS was disappointing. Further studies should examine other potentially active agents and/or combinations for this challenging clinical problem.

(C) 2011 Elsevier Inc. All rights reserved.

Address for correspondence: Rachel A. Freedman, MD, MPH, Dana-Farber Cancer Institute, Department of Medical Oncology, 44 Binney Street, Boston, MA 02115 Fax: 617-632-1930; rafreedman@ partners.org.

Disclosure: All authors report that they have no relevant relationships to disclose. 


\section{Keywords}

Central nervous system; Epothilone; Metastases; ZK-EPO

\section{Introduction}

Approximately $10 \%$ to $15 \%$ of women with metastatic breast cancer will experience brain metastases. ${ }^{1-6}$ Patients with human epidermal growth factor receptor 2-positive (HER2 ${ }^{+}$) or triple-negative breast cancer have a particularly high risk of central nervous system (CNS) involvement. ${ }^{6-10} \mathrm{Few}$ prospective trials have evaluated the role of systemic therapies for the treatment of brain metastases from breast cancer. ${ }^{11-14}$ Consequently there is no consensus on appropriate treatment for women in whom CNS progression develops after first-line CNS-directed therapy with whole-brain radiation therapy (WBRT), stereotactic radiosurgery (SRS), surgical resection, or a combination of modalities.

Epothilones are a new class of tubulin-stabilizing agents that have demonstrated responses in taxane-naive and pretreated breast cancer patients with systemic metastases. ${ }^{15-18}$ Sagopilone (ZK-Epothilone, ZK 219477, ZK-EPO), is an epothilone B analogue with activity in multidrug resistant (MDR)-displaying, taxane-resistant cell lines. ${ }^{19-21}$ Sagopilone is not a substrate of P-glycoprotein and readily crosses the blood-brain barrier. ${ }^{22,23}$ Furthermore, initial studies with sagopilone in patients with recurrent glioma demonstrated promising clinical activity. ${ }^{24,25}$ Although several phase $\mathrm{I}^{20,26,27}$ and phase $\mathrm{II}^{24,28}$ studies with sagopilone have been completed in multiple solid tumor settings, no studies have examined this agent's efficacy in breast cancer metastatic to the CNS.

We conducted a 2-stage phase II single-arm study to evaluate the clinical efficacy and toxicity profile of sagopilone in women with breast cancer and CNS metastases. Because of sagopilone's ability to cross the blood-brain barrier and evidence of systemic activity in treatment-refractory settings, we hypothesized that this agent would be active in patients with brain metastases. We report the findings from our study here.

\section{Patients and Methods}

\section{Study Cohort: Eligibility}

Adult patients aged $\geq 18$ years with histologically confirmed invasive breast cancer and progressive measurable CNS disease (defined as $\geq 1$ lesion $\geq 10 \mathrm{~mm}$ in longest dimension) were eligible. Previous CNS-directed therapy for brain metastases was required and could include surgery, WBRT, SRS, or a combination of therapies. Patients with progressive or new CNS lesions after CNS-directed therapy were eligible. Patients with multiple CNS lesions, 1 or several of which were previously treated with SRS or surgery, were eligible provided that they had $\geq 1$ residual untreated measurable lesion. Other inclusion criteria included a lack of increase in corticosteroid use during the week before baseline imaging, Eastern Cooperative Oncology Group (ECOG) performance status 0-2, and adequate bone marrow and end-organ function.

Patients were excluded if they had received chemotherapy within 3 weeks of study entry. Concurrent receipt of other investigational agents or cancer-directed therapy was not allowed, with the exception of ovarian suppression (when used for $>6$ months, during which time CNS disease progression was documented) and bisphosphonates. Patients were excluded if they received previous treatment with investigational chemotherapy for brain metastases, a previous epothilone, or concurrent enzyme-inducing antiepileptic drugs. Those 
with grade 2 or higher peripheral neuropathy, leptomeningeal disease as the only site of CNS disease, or $>2$ seizures during the month before study entry were not eligible.

This study was conducted in accordance with established guidelines as recommended by the US Department of Health and Human Services and was approved by the Institutional Review Board at Dana-Farber Cancer Institute/Harvard Cancer Center (Boston, MA).

\section{Treatment Plan and Stopping Rules}

In this 2-stage phase II single-arm study, the first patient cohort received a starting dose of sagopilone at $16 \mathrm{mg} / \mathrm{m}^{2}$ intravenously every 21 days. In August 2008, after 9 patients had been enrolled, the protocol was amended to evaluate sagopilone at $22 \mathrm{mg} / \mathrm{m}^{2}$ based on preliminary data from other breast cancer studies that the higher dose may be more efficacious (Lars Breimer, personal communication). The second cohort therefore had a starting dose of sagopilone $22 \mathrm{mg} / \mathrm{m}^{2}$ every 21 days. This protocol had early stopping rules for both toxicity ( $\geq 3$ patients [among the first 8] enrolled at $16 \mathrm{mg} / \mathrm{m}^{2}$ having grade $3 / 4$ CNS toxicity, $\geq 3$ patients enrolled at $22 \mathrm{mg} / \mathrm{m}^{2}$ withdrawing from the study before the first restaging because of toxicity) and for objective response (no responses in the first 12 patients on the $16 \mathrm{mg} / \mathrm{m}^{2}$ dose). If these stopping rules were not invoked, a total of 37 patients were to receive the $22 \mathrm{mg} / \mathrm{m}^{2}$ dose. If 4 of these 37 patients had an objective response, sagopilone would be considered worthy of further study.

At baseline and every 2 cycles, patients underwent brain magnetic resonance imaging (MRI) and computed tomography of the chest, abdomen, and pelvis. In addition, at baseline and at 6 weeks correlative imaging was obtained using modified high-resolution magnetic resonance angiography (MRA). Adverse events were captured at each visit. Tumorassociated neurologic signs and symptoms, corticosteroid use, and drug-related neurologic toxicities were documented each cycle using a worksheet designed for this study. Patients were continued on therapy until disease progression (CNS, systemic, or both), intercurrent illness that prevented therapy administration, unacceptable adverse events, or patient/ provider decision for withdrawal.

\section{Response Definitions and Statistical Analyses}

The primary efficacy endpoint was objective response rate (ORR, defined as complete response [CR] plus partial response [PR]) in the CNS. CNS responses were defined as follows: (1) CR, disappearance of all target and nontarget lesions; (2) PR, $250 \%$ decrease in the sum of volume of up to 5 target lesions, taking as reference the baseline sum volume; (3) progressive disease (PD), $\geq 40 \%$ increase in the sum of the volume of target lesions (relative to the smallest sum recorded since treatment started) or the appearance of $\geq 1$ new lesions; and (4) stable disease (SD), neither sufficient shrinkage nor increase to qualify for PRor PD. Evaluation of all CNS imaging was performed centrally at the Tumor Imaging Metrics Core (TIMC) of Dana-Farber/Harvard Cancer Center, as described previously. ${ }^{13}$ In non-CNS sites, responses were evaluated by individual site personnel (without central review) according to Response Evaluation Criteria in Solid Tumors (RECIST) 1.0. ${ }^{29}$

Secondary objectives included determination of safety and tolerability, ORR in non-CNS sites, clinical benefit rate (CR, PR, or SD in the CNS $\geq 24$ weeks with at least concurrent SD in non-CNS sites), first site of treatment failure, PFS, and OS. PFS and OS were analyzed using Kaplan-Meier product limit methods and the remaining secondary outcomes were summarized with descriptive statistics. 


\section{Correlative Imaging Studies}

Previous studies have suggested that CNS vessel tortuosity is provoked by malignancy and that normalization of vessels is associated with treatment response, even to agents without intrinsic antiangiogenic activity. ${ }^{30-33} \mathrm{We}$ therefore incorporated novel exploratory imaging analyses at baseline and at 6 weeks to characterize vessel density and tortuosity and to calculate the malignancy probability (MP) score using modified, high-resolution MRA, using methods previously described by Bullitt et al and Parikh et al; MP scores > 50 have been associated with malignancy. ${ }^{30-34}$

\section{Results}

\section{Patient Characteristics}

A total of 15 women were enrolled from August 1, 2007 to October 29, 2009 (9 patients at $16 \mathrm{mg} / \mathrm{m}^{2} ; 6$ patients at $\left.22 \mathrm{mg} / \mathrm{m}^{2}\right)$. Enrollment was terminated prematurely because of a combination of review of preliminary efficacy data and slow accrual. Baseline characteristics are shown in Table 1. The median age was 51 years (range, 31-74 years). Sixty percent of patients had a baseline ECOG performance status of 1 . Eleven patients had HER $2^{+}$tumors; only 1 patient had triple-negative disease. Approximately half of patients had received $\geq 3$ lines of chemotherapy for metastatic disease. All 15 patients had received previous WBRT; other previous CNS-directed treatments included surgery $(\mathrm{n}=2)$ and SRS $(\mathrm{n}=11)$.

\section{Efficacy}

The primary endpoint was CNS ORR. Of 15 patients, 2 achieved a PR (ORR, 13.3\%), 1 at each dose level (Table 2); 1 patient had estrogen receptor (ER) /HER2 disease and the other patient had ER ${ }^{+} / \mathrm{HER} 2^{-}$disease. Both of these patients remained in the study for 6 cycles before withdrawing for CNS progression. Selected brain MRI images from the partial responders are shown in Figure 1. No minor responses were observed. Notably, 10/15 patients withdrew from the study after only 2 cycles, 8 because of CNS progression, with a median duration in the study of 2 cycles (range, 1-6). Overall, reasons for withdrawing from the study were CNS progression $(n=12)$, toxicity $(n=1)$, physician/patient withdrawal for clinical progression $(\mathrm{n}=1)$, and death $(\mathrm{n}=1)$.

Results of analyses for secondary endpoints included ORR in non-CNS sites (0\%), clinical benefit rate (13.3\%), and 6-month PFS (0\%). Median PFS and OS were 1.4 months and 5.3 months, respectively. Three patients remained alive at last known follow-up. Of note, confidence intervals could not be calculated for ORR, OS, and PFS because of stopping rules based on number of responses or on number of patients completing 2 cycles of therapy, and also because neither dose cohort completed the planned accrual to the first stage.

\section{Toxicity}

Both dosing levels were generally well tolerated (Table 3), although 1 patient who received $22 \mathrm{mg} / \mathrm{m}^{2}$ experienced grade 4 transaminitis and grade 5 pulmonary embolus (PE) during the first cycle of therapy. The PE resulted in death from respiratory failure within 12 hours of emergency room presentation. Another patient withdrew from the study for unacceptable toxicity related to hydrocephalus; however this patient died of clinical progression shortly thereafter and the event was ultimately felt to be unrelated to therapy. Grade 3 adverse events were relatively uncommon at both dosing levels but were observed with higher frequency at the $22 \mathrm{mg} / \mathrm{m}^{2}$ dose. These events included fatigue $(\mathrm{n}=3)$, lymphopenia $(\mathrm{n}=3)$, leukopenia $(\mathrm{n}=2)$, muscle/joint aches, neuropathy, ataxia, dizziness, neutropenia, and weakness (1 patient each). Of note, the neuropathy and ataxia occurred in patients who received 1 to 2 cycles of therapy before clinical progression. Overall, 2 patients required 
dose reductions from $22 \mathrm{mg} / \mathrm{m}^{2}$ to $16 \mathrm{mg} / \mathrm{m}^{2}$ and 1 of these patients required an additional dose reduction to $12 \mathrm{mg} / \mathrm{m}^{2}$.

\section{Correlative Imaging Studies}

Of 15 patients who had baseline imaging, 10 had markedly abnormal intracranial vessel tortuosity (mean MP score, 99.2; range, 90-100). Of 10 evaluable patients with paired, posttreatment images at 6 weeks, all had persistently abnormal vasculature (MP score range, 99-100; maximum change in score from baseline $=1$ point). Other measures of vessel tortuosity (ie, sum of angles metric and inflection count metric) also demonstrated high levels of tortuosity for all evaluable patients (data not shown). Of note, the 2 patients with volumetric decreases in target lesions both had persistently and highly abnormal tortuosity values (MP score, 100 at both time points for both patients). Example images illustrating the observed vessel tortuosity in 1 patient are shown in Figure 2.

\section{Neurologic Signs and Symptoms}

Neurologic examination worksheets were completed by providers at baseline and at each visit; they evaluated patients for several domains: level of consciousness (LOC), symptoms (ie, headaches, nausea), cranial nerve deficits, language, sensation, ataxia, overall neurologic status (improved, stable, worsening), and potential attributions to therapy. At baseline all patients were reported to have a normal LOC; new somnolence was recorded in 2 patients at progression. Headaches, dizziness, and vertigo were infrequent and were generally mild to moderate with the exception of 1 patient who had severe dizziness at progression (felt to be related to therapy). Two patients had moderate ataxia and 5 patients had mild ataxia attributed to disease progression; 1 patient could not be assessed for ataxia because of bedridden status from disease progression. It is of note that ataxia was more commonly reported on the worksheets than on toxicity assessments from medical record review.

With regard to findings from the neurologic worksheets for the 2 responders, 1 partial responder remained asymptomatic until progression; the second responder had begun sagopilone while on a stable dose of dexamethasone (4 mg daily). These steroids were discontinued by the start of cycle 2 but were then restarted at progression. Other neurologic signs and symptoms were stable while these patients were receiving therapy. Changes in overall neurologic symptoms and signs for all women in the study are shown in Table 4. Overall worsening neurologic status was noted in 9 patients at progression; 7 of these patients also had clinical worsening in $\geq 1$ domain (included ataxia in all 7 patients). Five patients had overall worsening neurologic signs and symptoms that were felt to be at least partially attributed to sagopilone. These observations are somewhat challenging to interpret because of concurrent disease progression.

\section{Premature Trial Closure}

Over the course of our study several concerns arose, which resulted in a decision to close the protocol after 15 patients had enrolled (reaching the time when stopping rules could be assessed). Emerging data from other ongoing studies showed that the activity of sagopilone was less than expected among breast cancer patients in general. ${ }^{35}$ In addition, these 15 patients experienced moderate toxicity, and a majority of patients had rapid progression while in the study. Because of these issues as well as slow accrual, we felt that continuation of the study as planned was not an appropriate use of patient resources.

\section{Discussion}

In this phase II study we evaluated the efficacy and toxicity profile of sagopilone in patients with breast cancer and progressive brain metastases. We observed a CNS ORR of $13.3 \%$ 
with a median PFS of 1.4 months and median OS of 5.3 months. Two thirds of patients withdrew from the study after the first restaging evaluation, mostly for progressive CNS disease, and most patients had worsening neurologic status at the time of progression. Sagopilone toxicities were largely manageable. The most common grade 3 toxicities were lymphopenia and fatigue. In a previous phase I study with this agent (306821) (Sagopilone [ZK-EPO, ZK219477] Investigator's Brochure, Version 6.0, November 28, 2007, Bayer Schering Pharma AG), 2 cases of ataxia of CNS origin were observed, 1 at the $22 \mathrm{mg} / \mathrm{m}^{2}$ dose and 1 at the $29 \mathrm{mg} / \mathrm{m}^{2}$ dose, raising concerns about the safety profile of sagopilone (Investigator Brochure). Reassuringly we did not observe any definite cases of central ataxia that were clearly attributable to therapy.

The advent of novel therapies has been associated with prolongation of survival for some women with CNS metastases, ${ }^{36,37}$ likely as a result of better systemic disease control. Our trial may be representative of these outcomes, as the median time from diagnosis of CNS metastasis to study entry was 25 months (range, 6-52 months). Furthermore nearly all patients withdrew from the study for CNS (as opposed to systemic) progression and 3 (20\%) patients remained alive at 1.5 to 2.5 years after study registration. Investigation of CNSdirected therapies will become increasingly important as a number of these long-term survivors have the brain as their dominant site of disease progression.

Although preclinical studies demonstrated sagopilone's ability to cross the blood-brain barrier ${ }^{23}$ results of clinical trials with this drug were somewhat disappointing. This may have occurred for several reasons. When we initiated this study, preliminary evidence of antitumor activity had been observed across multiple early-phase trials of sagopilone in solid tumors, including breast cancer. In addition, prolonged responses were observed in glioblastoma multiforme (GBM) ${ }^{24}$ Since then a subsequent phase II trial of sagopilone in 38 patients with recurrent GBM demonstrated no objective disease responses, and the authors concluded that sagopilone was not worthy of future study for this indication. ${ }^{38}$ Furthermore in a study of 65 women with refractory metastatic breast cancer without symptomatic CNS involvement, the ORRfor 2 dosing levels was $4.6 \%$ and the median number of cycles delivered was $2 .{ }^{35}$

These evolving data have demonstrated that sagopilone may simply not be an adequately effective agent to treat breast cancer, as suggested by the low systemic response rates in women with metastatic disease outside the CNS. Results of these trials also contributed to our decision to close the current study before full accrual. However other epothilones are still under study in women with breast cancer metastatic to the brain. For example in a preliminary report of 38 evaluable women with progressive brain metastases who received patupilone, a natural epothilone B, the 3-month PFS was $37 \%$ and the median CNS PFS was 57 days. Five (13\%) women experienced PR in the CNS and 12 women (32\%) had SD, meeting patupilone's prespecified threshold for further investigation. ${ }^{39}$ In our study, although the 2 responders remained in the study for 6 cycles, no additional patients achieved $\mathrm{SD}>12$ weeks.

Because the majority of patients enrolled in this study had HER2 ${ }^{+}$disease, it is conceivable that we would have observed more frequent and/or more durable responses with sagopilone if administered in combination with a HER2-targeted agent. For example the addition of lapatinib to capecitabine in patients with HER2 ${ }^{+}$metastatic breast cancer was associated with improvements in ORR and PFS in addition to a nonsignificant decrease in CNS events. ${ }^{40}$ Because of the limited observed activity of sagopilone in women with metastatic breast cancer (with and without CNS disease), further study of sagopilone in this patient population will not likely not be pursued. 
Our study had several limitations. This was a small single-center study of heavily pretreated patients. It is possible that we would have observed more activity in patients who had received less chemotherapy exposure previously; however such patients are also less likely to have refractory brain metastases, which was the subject of our trial. All patients with measurable metastatic CNS disease were eligible for this trial irrespective of tumor subtype, which may have limited our ability to detect a benefit in a specific subset of breast cancer patients. However the 2 women who experienced PRs had ER ${ }^{+} / \mathrm{HER}^{-}$and $\mathrm{ER}^{+} / \mathrm{HER} 2^{+}$ primary breast cancers, which does not suggest a clear trend for activity in a particular subtype. Only 1 patient in our study had triple-negative disease (and had disease progression at first re-staging). Although it is possible that a clinical benefit was missed by prematurely closing our trial after enrollment of 15 patients, in the context of emerging data of this agent in systemic metastatic breast cancer, we feel that our decision was justified. Although we did not include formal neurocognitive testing as a measure of clinical benefit, we found that the neurologic signs and symptoms worksheet was sensitive to change, as most patients experienced worsening symptoms (ie, ataxia) at progression. Finally with respect to the correlative imaging, we cannot rule out the possibility that significant vessel changes (in responders) may have occurred beyond 6 weeks; whether vessel changes are a true marker of clinical benefit of sagopilone is unknown.

The results of our study have demonstrated limited clinical activity of sagopilone in patients with breast cancer metastatic to the brain. Further study of sagopilone as a single agent in this patient population is not currently warranted. We cannot rule out the possibility that responses in HER2 ${ }^{+}$patients may have been more frequent and/or more durable with concurrent HER2-directed therapy; however there may be more attractive chemotherapy partners. Future investigations of novel regimens for women with brain metastases are urgently needed and should be a priority for research.

\section{Conclusion}

Patients with progressive brain metastases from breast cancer have limited treatment options. Few prospective trials have evaluated the role of systemic therapies for this challenging clinical situation, and consequently there is no consensus on appropriate treatment for women who experience progression after first-line CNS-directed therapy. In addition to preclinical data demonstrating sagopilone's ability to cross the blood-brain barrier, preliminary reports suggested promising systemic activity of sagopilone for patients with stage IV breast cancer and for those with GBM. These preliminary data provided the rationale for our study design.

We conducted a phase II study of sagopilone, an epothilone B analogue, in patients with breast cancer brain metastases that progressed after receipt of first-line CNS-directed therapy. Women received sagopilone at $16 \mathrm{mg} / \mathrm{m}$ or $22 \mathrm{mg} / \mathrm{m}^{2}$ intravenously every 21 days. Our primary endpoint was CNS ORR, and secondary end-points included toxicity, PFS, and OS. Using modified high-resolution MRA, we also evaluated changes in vessel tortuosity with treatment. Among the 15 women enrolled in the study, 2 patients achieved a PR (ORR $13.3 \%$ ) and remained in the study for 6 cycles. Responses were not associated with normalization of tumor-associated vessels on correlative imaging studies. Median PFS and OS were 1.4 months and 5.3 months, respectively, and the most common grade 3 toxicities were lymphopenia and fatigue. Enrollment was stopped prematurely because of limited observed activity, evolving data regarding the lack of activity in metastatic breast cancer and glioblastoma, and slow accrual. 
The results of our study were disappointing and further study of sagopilone as a single agent in this patient population is not currently warranted. Future investigations of novel regimens for women with brain metastases are urgently needed and should be a priority for research.

\section{Acknowledgments}

We would like to thank Trinity Urban at the Tumor Imaging Metrics Core for her assistance with central radiology review and Yao Wang and Marius Giurescu at Bayer for their thoughtful collaboration and support.

This work was supported by the American Society of Clinical Oncology Career Development Award (Nancy Lin), the National Cancer Institute Specialized Program of Research Excellence in Breast Cancer at Dana-Farber/Harvard Cancer Center (CA89393), the Karen Webster and David Evans Research Fund (Nancy Lin, Eric Winer); the Breast Cancer Research Foundation (Nancy Linn)the Dana-Farber/Harvard Cancer Center grant CA006516 (Lixian Sun, Rebecca Gelman), the National Institutes of Health (R01 CA124608 and R01 EB000219) [E.B.], and Bayer HealthCare Pharmaceuticals.

\section{References}

1. Tsukada Y, Fouad A, Pickren JW, et al. Central nervous system metastasis from breast carcinoma. Autopsy study Cancer. 1983; 52:2349-54.

2. Patanaphan V, Salazar OM, Risco R. Breast cancer: metastatic patterns and their prognosis. South Med J. 1988; 81:1109-12. [PubMed: 3420442]

3. Barnholtz-Sloan JS, Sloan AE, et al. Incidence proportions of brain metastases in patients diagnosed (1973 to 2001) in the Metropolitan Detroit Cancer Surveillance System. J Clin Oncol. 2004; 22:2865-72. [PubMed: 15254054]

4. Engel J, Eckel R, Aydemir U, et al. Determinants and prognoses of locoregional and distant progression in breast cancer. Int J Radiat Oncol Biol Phys. 2003; 55:1186-95. [PubMed: 12654426]

5. Niwinska A, Murawska M, Pogoda K. Breast cancer brain metastases: differences in survival depending on biological subtype, RPA RTOG prognostic class and systemic treatment after wholebrain radiotherapy (WBRT). Ann Oncol. 2010; 21:942-8. [PubMed: 19840953]

6. Lin NU, Bellon JR, Winer EP. CNS metastases in breast cancer. J Clin Oncol. 2004; 22:3608-17. [PubMed: 15337811]

7. Bendell JC, Domchek SM, Burstein HJ, et al. Central nervous system metastases in women who receive trastuzumab-based therapy for metastatic breast carcinoma. Cancer. 2003; 97:2972-77. [PubMed: 12784331]

8. Lin NU, Claus E, Sohl J, et al. Sites of distant recurrence and clinical outcomes in patients with metastatic triple-negative breast cancer: high incidence of central nervous system metastases. Cancer. 2008; 113:2638-45. [PubMed: 18833576]

9. Pestalozzi BC. Brain metastases and subtypes of breast cancer. Ann Oncol. 2009; 20:803-5. [PubMed: 19403934]

10. Kennecke H, Yerushalmi R, Woods R, et al. Metastatic behavior of breast cancer subtypes. J Clin Oncol. 28:3271-77. [PubMed: 20498394]

11. Franciosi V, Cocconi G, Michiara M, et al. Front-line chemotherapy with cisplatin and etoposide for patients with brain metastases from breast carcinoma, nonsmall cell lung carcinoma, or malignant melanoma: a prospective study. Cancer. 1999; 85:1599-1605. [PubMed: 10193952]

12. Rivera E, Meyers C, Groves M, et al. Phase I study of capecitabine in combination with temozolomide in the treatment of patients with brain metastases from breast carcinoma. Cancer. 2006; 107:1348-54. [PubMed: 16909414]

13. Lin NU, Carey LA, Liu MC, et al. Phase II trial of lapatinib for brain metastases in patients with human epidermal growth factor receptor 2-positive breast cancer. J Clin Oncol. 2008; 26:1993-9. [PubMed: 18421051]

14. Lin NU, Dieras V, Paul D, et al. Multicenter phase II study of lapatinib in patients with brain metastases from HER2-positive breast cancer. Clin Cancer Res. 2009; 15:1452-9. [PubMed: 19228746] 
15. Roche H, Yelle L, Cognetti F, et al. Phase II clinical trial of ixabepilone (BMS-247550), an epothilone B analog, as first-line therapy in patients with metastatic breast cancer previously treated with anthracycline chemotherapy. J Clin Oncol. 2007; 25:3415-20. [PubMed: 17606972]

16. Lin N, Brakora K, Seiden M. BMS-247550 Bristol-Myers Squibb/GBF. Curr Opin Investig Drugs. 2003; 4:746-56.

17. Thomas E, Tabernero J, Fornier M, et al. Phase II clinical trial of ixabepilone (BMS-247550), an epothilone B analog, in patients with taxane-resistant metastatic breast cancer. J Clin Oncol. 2007; 25:3399-406. [PubMed: 17606975]

18. Rivera E, Lee J, Davies A. Clinical development of ixabepilone and other epothilones in patients with advanced solid tumors. Oncologist. 2008; 13:1207-23. [PubMed: 19088324]

19. Strube A, Hoffmann J, Stepina E, et al. Sagopilone inhibits breast cancer bone metastasis and bone destruction due to simultaneous inhibition of both tumor growth and bone resorption. Clin Cancer Res. 2009; 15:3751-9. [PubMed: 19470728]

20. Galmarini CM. Sagopilone, a microtubule stabilizer for the potential treatment of cancer. Curr Opin Investig Drugs. 2009; 10:1359-71.

21. Klar U, Hoffmann J, Giurescu M. Sagopilone (ZK-EPO): from a natural product to a fully synthetic clinical development candidate. Expert Opin Investig Drugs. 2008; 17:1735-48.

22. Hoffmann J, Vitale I, Buchmann B, et al. Improved cellular pharmacokinetics and pharmacodynamics underlie the wide anticancer activity of sagopilone. Cancer Res. 2008; 68:5301-8. [PubMed: 18593931]

23. Hoffmann J, Fichtner I, Lemm M, et al. Sagopilone crosses the blood-brain barrier in vivo to inhibit brain tumor growth and metastases. Neuro Oncol. 2009; 11:158-66. [PubMed: 18780814]

24. Silvani A, Gaviani P, Fiumani A, et al. Systemic sagopilone (ZK-EPO) treatment of patients with recurrent malignant gliomas. J Neurooncol. 2009; 95:61-4. [PubMed: 19381446]

25. Peereboom DM, Supko JG, Carson KA, et al. A phase I/II trial and pharmacokinetic study of ixabepilone in adult patients with recurrent high-grade gliomas. J Neurooncol. 2010; 100:261-8. [PubMed: 20449631]

26. Arnold D, Voigt W, Kiewe P, et al. Weekly administration of sagopilone (ZK-EPO), a fully synthetic epothilone, in patients with refractory solid tumours: results of a phase I trial. Br J Cancer. 2009; 101:1241-7. [PubMed: 19773753]

27. Schmid P, Kiewe P, Possinger K, et al. Phase I study of the novel, fully synthetic epothilone sagopilone (ZK-EPO) in patients with solid tumors. Ann Oncol. 2010; 21:633-9. [PubMed: 19880436]

28. Daud A, Weber J, Urbas P, et al. Phase II trial of sagopilone (ZK-EPO), a novel synthetic epothilone, with significant activity in metastatic melanoma. J Clin Oncol. 2009; 27(suppl):15S. (abstract 9031). Available at http://www.asco.org.

29. Therasse P, Arbuck SG, Eisenhauer EA, et al. New guidelines to evaluate the response to treatment in solid tumors. European Organization for Research and Treatment of Cancer, National Cancer Institute of the United States, National Cancer Institute of Canada. J Natl Cancer Inst. 2000; 92:205-16. [PubMed: 10655437]

30. Bullitt E, Ewend MG, Aylward S, et al. Abnormal vessel tortuosity as a marker of treatment response of malignant gliomas: preliminary report. Technol Cancer Res Treat. 2004; 3:577-84. [PubMed: 15560715]

31. Bullitt E, Lin NU, Smith JK, et al. Blood vessel morphologic changes depicted with MR angiography during treatment of brain metastases: a feasibility study. Radiology. 2007; 245:82430. [PubMed: 17954616]

32. Bullitt E, Gerig G, Pizer SM, et al. Measuring tortuosity of the intracerebral vasculature from MRA images. IEEE Trans Med Imaging. 2003; 22:1163-71. [PubMed: 12956271]

33. Bullitt E, Zeng D, Gerig G, et al. Vessel tortuosity and brain tumor malignancy: a blinded study. Acad Radiol. 2005; 12:1232-40. [PubMed: 16179200]

34. Parikh AH, Smith JK, Ewend MG, et al. Correlation of MR perfusion imaging and vessel tortuosity parameters in assessment of intracranial neoplasms. Technol Cancer Res Treat. 2004; 3:585-90. [PubMed: 15560716] 
35. Morrow PH, Divers SG, Provencher L, et al. Phase II study of sagopilone (ZK-Epo) in patients with recurrent metastatic breast cancer (MBC). J Clin Oncol. 2009; 15s, 27(suppl):15S. (abstract 1083). Available at http://www.asco.org.

36. Eichler AF, Kuter I, Ryan P, et al. Survival in patients with brain metastases from breast cancer: the importance of HER-2 status. Cancer. 2008; 112:2359-67. [PubMed: 18361426]

37. Melisko ME, Moore DH, Sneed PK, et al. Brain metastases in breast cancer: clinical and pathologic characteristics associated with improvements in survival. J Neurooncol. 2008; 88:35965. [PubMed: 18398574]

38. Stupp R, Tosoni A, Taal W, et al. Phase II trial of the epothilone analog sagopilone (ZK219477; ZK EPO) in patients with recurrent glioblastoma: initial report of the EORTC study 26061. J Clin Oncol. 2008; 26(suppl) abstract 2015.

39. Murphy, C.; Nulsen, B.; Rump, M., et al. Phase II trial of patupilone in patients with breast cancer brain metastases progressing or recurring after whole brain radiotherapy; Abstract presented at: 2009 Breast Cancer Symposium; October 8-10, 2009; San Francisco, CA. Abstract 234. Available at http://www.asco.org

40. Geyer CE, Forster J, Lindquist D, et al. Lapatinib plus capecitabine for HER2-positive advanced breast cancer. N Engl J Med. 2006; 355:2733-43. [PubMed: 17192538] 


\section{Clinical Practice Points}

- Management of breast cancer that has metastasized to the brain is a challenging clinical problem, particularly for those patients who experience recurrent CNS disease.

- Current treatment options for these patients may include repeated stereotactic surgery, whole brain radiation therapy, craniotomy, systemic therapy, or enrollment on a clinical trial.

- The results of our phase II protocol of sagopilone in women with breast cancer and progressive brain metastases suggest minimal and short-lived activity of this agent with regard to central nervous system response.

- Further study of sagopilone as a single agent in this setting is not currently warranted. Future investigations of novel treatments for this clinical problem are urgently needed and should be a priority for research. 


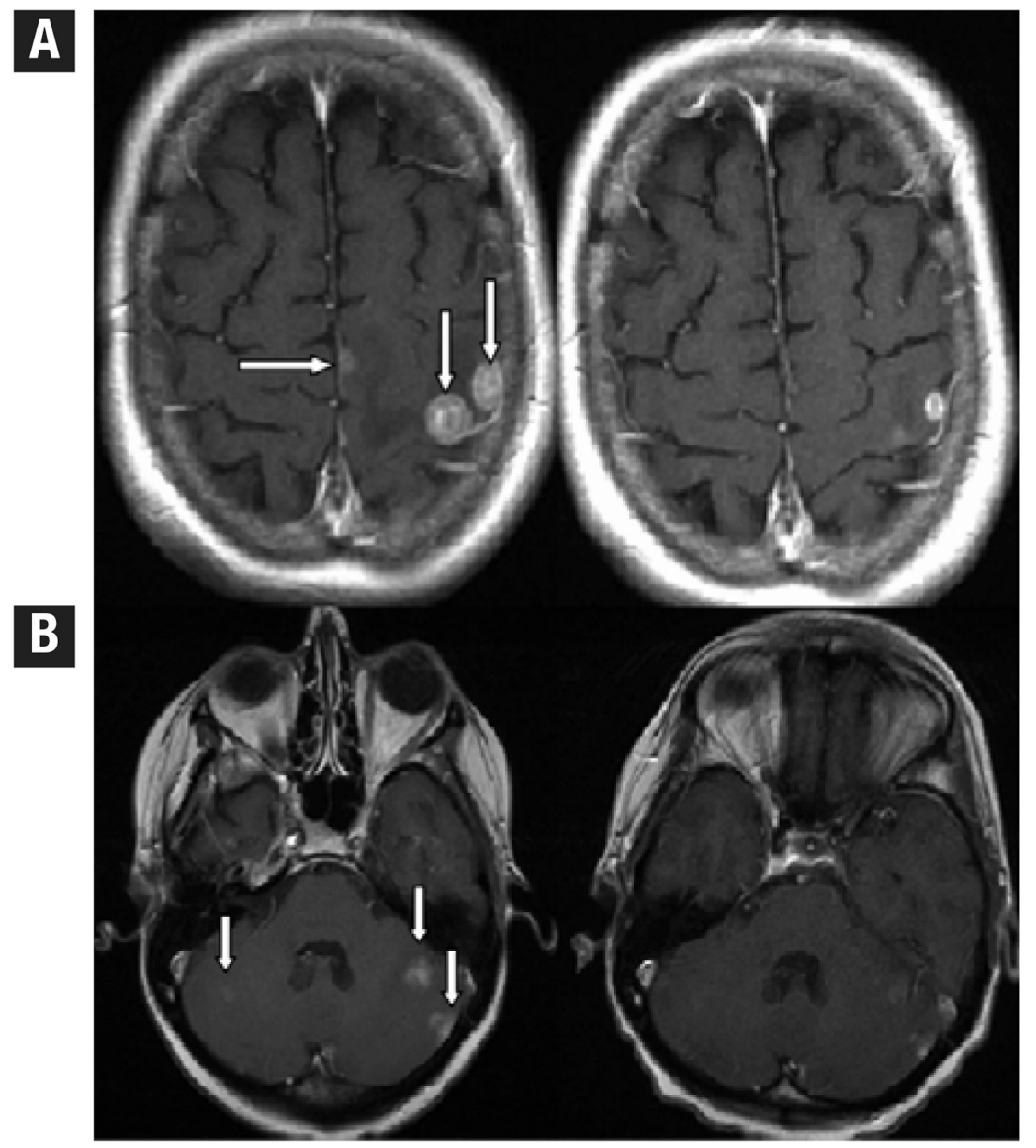

Figure 1.

Selected Images for Partial Responders. (A) Comparable Axial T1-GAD Slices at Times 1 and 2 in Patient 5 With Multiple Lesions. Arrows Point to 2 Large Peripheral and a Third Small Midline Lesion at Time 1. At Time 2, All 3 Lesions Reduced Dramatically in Volume as Illustrated Here in the 2-Dimensional Slice Data; (B) Comparable Axial T1-GAD Slices at Times 1 and 2 in Patient 11 With Multiple Lesions. Arrows Point to 2 Left Cerebellar and a Faint Right Cerebellar Lesion. At Time 2, All 3 Lesions Reduced Dramatically in Volume as Illustrated Here in the 2-Dimensional Slice Data 

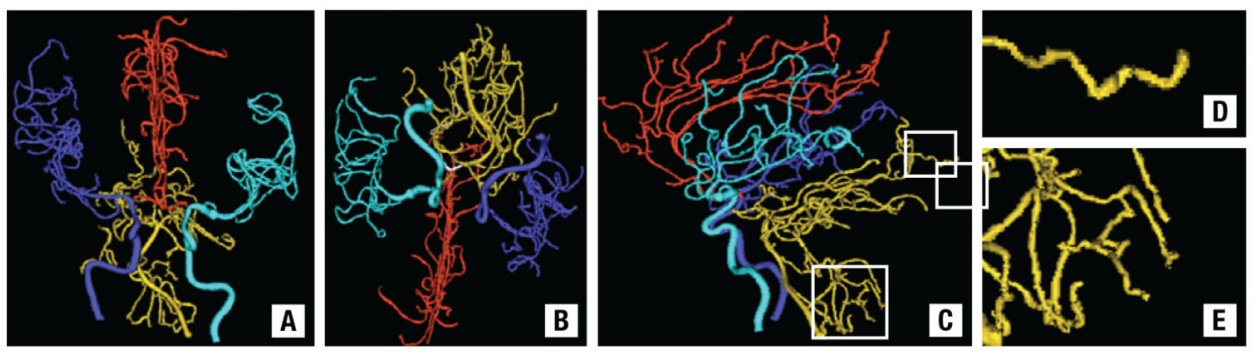

Figure 2.

Images of 3-Dimensional Segmented Vessels From a Patient in the Study. Connected Vessel Groups Have Been Color-Coded for Easier Identification. Cyan = Left Middle Cerebral Group, Blue = Right Middle Cerebral Group, Red = Anterior Cerebral Group, Gold = Posterior Cerebral Group. (A) AP View; (B) Base View; (C) Lateral View. White Boxes Indicate Regions Enlarged in D and E. (D and E) Magnifications of Part of the Posterior Circulation Shown in C to Illustrate the Abnormal Vessel Tortuosity Associated With Cancer 
Table 1

Patient Characteristics $(\mathrm{N}=15)$

\begin{tabular}{|c|c|}
\hline Characteristic & No. of Patients (\%) \\
\hline \multicolumn{2}{|l|}{ Age, Years } \\
\hline Median & 51 years \\
\hline Range & 31-74 years \\
\hline White Race & $15(100)$ \\
\hline Female Gender & $15(100)$ \\
\hline \multicolumn{2}{|l|}{ ECOG Performance Status } \\
\hline 0 & $2(13)$ \\
\hline 1 & $9(60)$ \\
\hline 2 & $4(27)$ \\
\hline \multicolumn{2}{|l|}{ Stage at Initial Diagnosis } \\
\hline I & $5(33)$ \\
\hline II & $5(33)$ \\
\hline III & $1(7)$ \\
\hline IV & $4(27)$ \\
\hline \multicolumn{2}{|l|}{ Primary Tumor Receptor Status } \\
\hline $\mathrm{ER}^{+}, \mathrm{HER} 2^{-}$ & $3(20)$ \\
\hline $\mathrm{ER}^{+}, \mathrm{HER} 2^{+a}$ & $7(47)$ \\
\hline $\mathrm{ER}^{-}, \mathrm{HER} 2^{+a}$ & $4(27)$ \\
\hline Triple negative & $1(7)$ \\
\hline \multicolumn{2}{|c|}{ Adjuvant Chemotherapy Received } \\
\hline Anthracycline & $3(20)$ \\
\hline Anthracycline and taxane & $4(27)$ \\
\hline Nonanthracycline, nontaxane & $0(0)$ \\
\hline Trastuzumab-inclusive & $2(13)$ \\
\hline None & $4(27)$ \\
\hline Not Applicable $b$ & $4(27)$ \\
\hline \multicolumn{2}{|c|}{ Number Of Metastatic Disease Sites } \\
\hline Median & 3 \\
\hline Range & $1-5$ \\
\hline \multicolumn{2}{|l|}{ Sites of Disease } \\
\hline CNS & $15(100)$ \\
\hline Lung or pleura & $6(40)$ \\
\hline Liver & $7(47)$ \\
\hline Bone & $10(67)$ \\
\hline Breast or chest wall & $0(0)$ \\
\hline Other sites & $4(27)$ \\
\hline
\end{tabular}




\begin{tabular}{|c|c|}
\hline Characteristic & No. of Patients (\%) \\
\hline \multicolumn{2}{|c|}{ Number of Previous Chemotherapy Regimens (for Metastases Only) } \\
\hline 0 & $2(13)$ \\
\hline 1 & $4(27)$ \\
\hline 2 & $2(13)$ \\
\hline 23 & $7(47)$ \\
\hline \multicolumn{2}{|c|}{ Systemic Therapy Exposure (for Metastases Only) } \\
\hline Taxane & $9(60)$ \\
\hline Capecitabine & $11(73)$ \\
\hline Trastuzumab or lapatinib & $9(60)$ \\
\hline Vinorelbine & $7(47)$ \\
\hline Carboplatin & $3(20)$ \\
\hline Temozolomide & $1(7)$ \\
\hline Hormonal therapy & $7(47)$ \\
\hline Antiangiogenic therapy & $0(0)$ \\
\hline Anthracycline & $1(7)$ \\
\hline \multicolumn{2}{|c|}{ Previous Treatment of CNS Disease } \\
\hline CNS surgery & $2(15)$ \\
\hline SRS brain only & $0(0)$ \\
\hline WBRT & $15(100)$ \\
\hline SRS and WBRT & $11(73)$ \\
\hline
\end{tabular}

Abbreviations: $\mathrm{CNS}=$ central nervous system; ECOG $=$ Eastern Cooperative Oncology Group; ER = estrogen receptor; HER2 = human epidermal growth factor receptor $2 ; \mathrm{SRS}=$ stereotactic radiosurgery; $\mathrm{WBRT}$ = whole brain radiotherapy.

${ }^{a}$ HER $2^{+}=$deemed positive by immunohistochemistry or fluorescence in situ hybridization.

$b_{\text {Initial diagnosis was stage IV disease. }}$ 
Table 2

Overall CNS Activity Rate for Sagopilone ( $\mathbf{N}=15)$

\begin{tabular}{l|l}
\hline Response & Number of Patients (\%) \\
\hline Overall Response & $2(13)$ \\
\hline Complete Response & $0(0)$ \\
\hline Partial Response & $2(13)$ \\
\hline Stable Disease (> 12 Weeks) & $0(0)$ \\
\hline Clinical Benefit Rate $b$ & $2(13)$ \\
\hline 6-Month PFS & $0(0)$ \\
\hline
\end{tabular}

Abbreviation: PFS = progression-free survival.

a Note: Confidence intervals could not be calculated because of the different stopping rules and because neither dose cohort completed the planned accrual to the first stage.

$b$

$b$ Defined as complete response, partial response, or stable disease $\geq 24$ weeks in the central nervous system, with at least concurrent stable disease in non-central nervous system sites. 


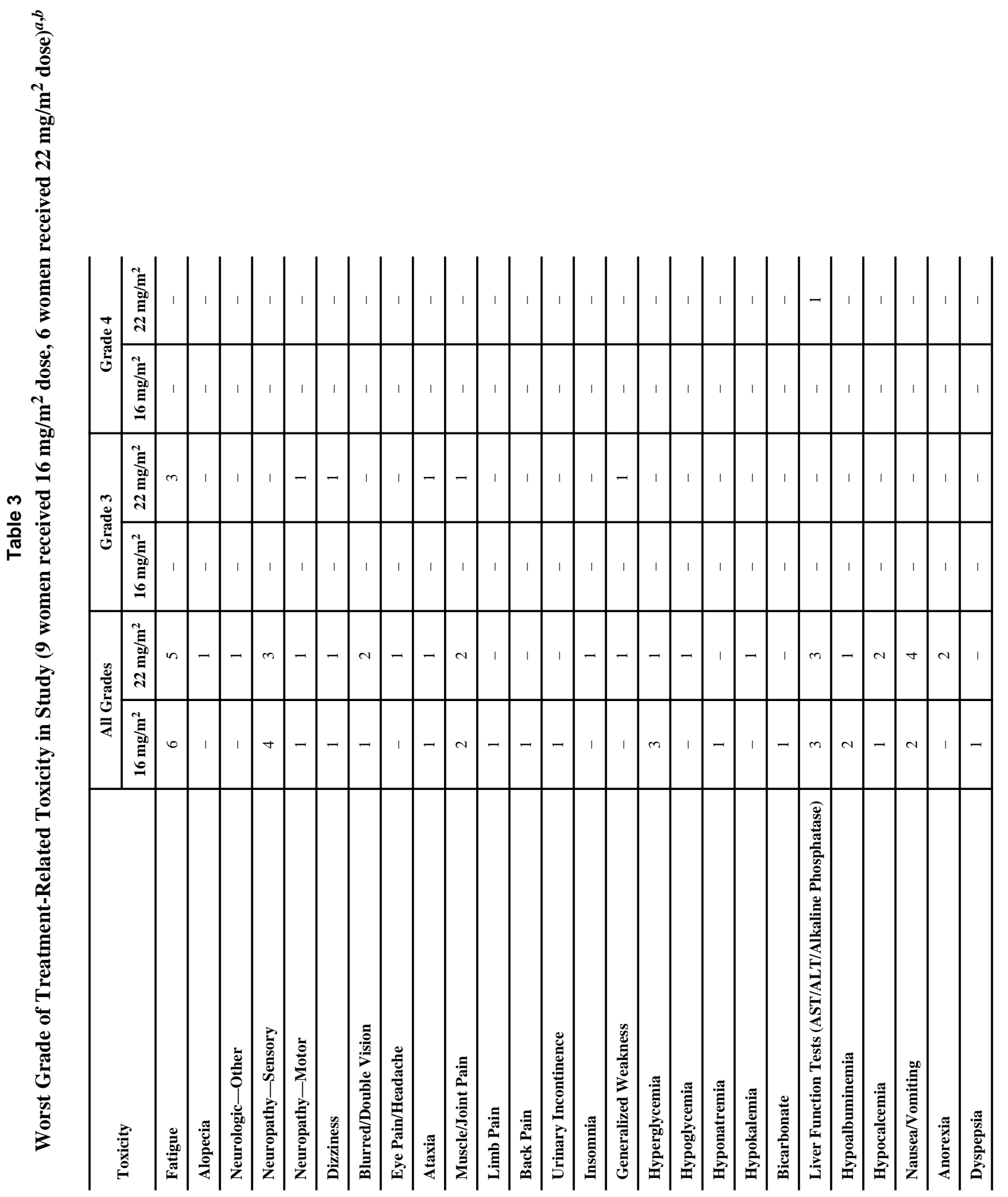




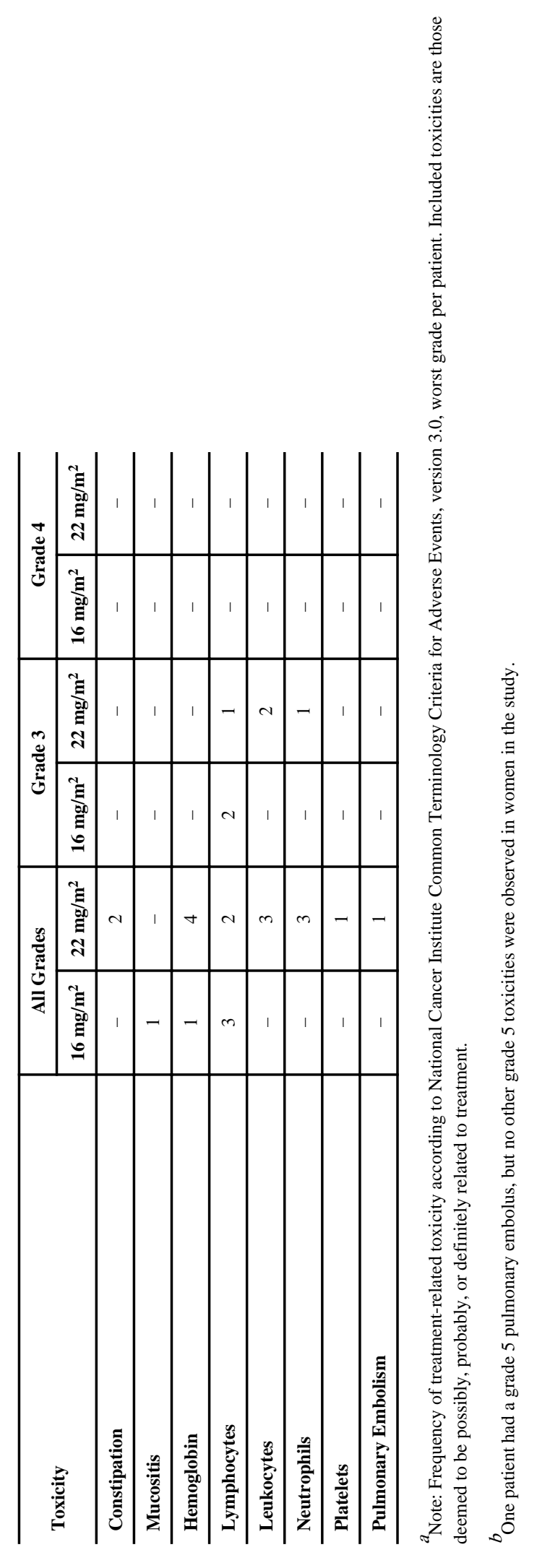


Table 4

\section{Changes in Neurologic Signs and Symptoms for Women Receiving Sagopilone $(\mathbf{N}=15)^{a}$}

\begin{tabular}{l|c|c|c|c}
\hline \multirow{2}{*}{ Study Time Point } & \multicolumn{2}{|c|}{ Overall Status of Neurologic Signs and Symptoms (n) } & \multirow{2}{*}{ Worsening of $\geq 1$ Neurologic Domain $b$} \\
\cline { 2 - 4 } & Improved & Stable & Worsening & 7 (of 11) \\
\hline Midtherapy & 1 & 8 & $2^{c}$ & 10 (of 14) $^{d}$ \\
\hline Progression/withdrew from study & 0 & 5 & $9^{c}$ & ${ }^{c}$ \\
\hline
\end{tabular}

a Three patients had progression at second assessment and did not have "midtherapy" evaluations; 1 patient did not have assessments beyond baseline.

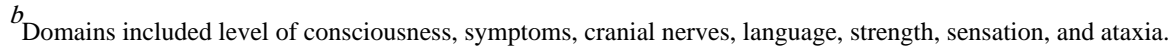

${ }^{c}$ At midtherapy and progression in 1 woman and 5 women, respectively, the signs and symptoms were felt to be possibly, probably, or definitely related to therapy.

$d_{\text {Seven of these patients had worsening overall status. }}$ 